\title{
Extremal limits of the Cvetič-Youm black hole and nilpotent orbits of $\mathrm{G}_{2(2)}$
}

\author{
Josef Lindman Hörnlund and Amitabh Virmani \\ Physique Théorique et Mathématique, \\ Université Libre de Bruxelles and International Solvay Institutes \\ Campus Plaine C.P. 231, B-1050 Bruxelles, Belgium \\ jlindman, avirmani@ulb.ac.be
}

\begin{abstract}
We study extremal cohomogeneity one five-dimensional asymptotically flat black holes of minimal supergravity in terms of the geodesics generated by nilpotent elements of the Lie algebra $\mathfrak{g}_{2(2)}$ on the coset manifold $\mathrm{G}_{2(2)} / \mathrm{SO}(2,2)$. There are two branches of regular extremal black holes with these properties: (i) the supersymmetric BMPV branch, and (ii) the non-supersymmetric extremal branch. We show that both of these branches are reproduced by nilpotent $\mathrm{SO}(2,2)$-orbits. Furthermore, we show that the partial ordering of nilpotent orbits of $\mathrm{G}_{2(2)}$ is in one-to-one correspondence with the phase diagram of these extremal black holes.
\end{abstract}




\section{Contents}

1 Introduction $\quad 2$

2 The Cvetič-Youm family and its extremal limits 3

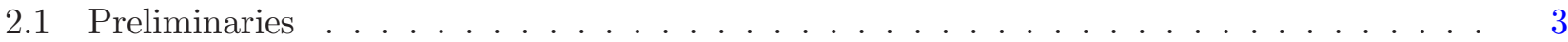

2.2 Cvetič-Youm, BMPV, and extremal non-supersymmetric black holes . . . . . . . . . 5

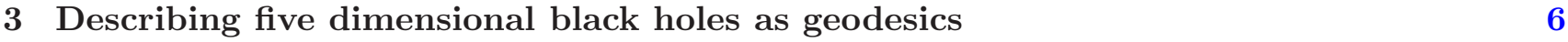

4 Nilpotent orbits and phases of extremal black holes $r$

4.1 Supersymmetric branch . . . . . . . . . . . . . . . . . . . . . . 10

4.2 Non-supersymmetric branch . . . . . . . . . . . . . . . . . . . . . . . . 11

5 Discussion $\quad 12$

$\begin{array}{lr}\text { A Cvetič-Youm metric } & 12\end{array}$

$\begin{array}{ll}\text { B Asymptotic reductive decomposition } & 13\end{array}$

\section{Introduction}

Asymptotically flat single center black holes of various four-dimensional supergravity theories have been extensively studied in the literature. At the classical level many physical and thermodynamical properties of these black holes are well understood. For a large number of theories single center fourdimensional black holes for any allowed configuration of charges can be explicitly constructed. This is particularly so for extremal black holes - BPS as well as non-BPS - where in a large number of cases a complete classification is already available. See for instance $[1,2,3,4,5,6,7,8,9,10,11,12,13$, 14, 15, 16, 17, 18, 19, 20] for recent discussions and references therein. Algebraic methods, see e.g., $[9,10,13,14,15,17]$, in terms of a three dimensional sigma-model with symmetric target space $G / \tilde{K}$ have proven to be extremely useful in this regard. These methods provide us with a systematic technique to find all extremal as well as non-extremal black holes. See [10, 17, 20] for detailed explicit calculations for the $N=2, D=4 \mathrm{~S}^{3}$ model. ( $\mathrm{S}^{3}$ model is obtained by setting $\mathrm{S}=\mathrm{T}=\mathrm{U}$ in the STU model.) Practical implementation of these methods may be technically challenging for supergravity theories of interest, but, there are no conceptual obstacles as to the applicability of these techniques. These developments have led to, among other things, a fairly complete understanding of the attractor mechanism for BPS as well as extremal non-BPS black holes [15].

It is natural to ask how much of these considerations extend to five-dimensional asymptotically flat black holes. Non-extremal five-dimensional black holes have been explored in various contexts from the three-dimensional sigma-model point of view [21, 22, 23, 24, 25, 26, 10, 27], but such studies have not yet been adapted to extremal black holes. Reference [28] took some steps in this direction. From the sigmamodel perspective, the difference between extremal and non-extremal black holes arises due to the fact that the relevant orbits for extremal black holes are nilpotent $\tilde{K}$-orbits of the three-dimensional hidden 
symmetry group, where as for non-extremal black holes the relevant orbits are semi-simple ones. Berkooz and Pioline in reference [28] studied supersymmetric five-dimensional black holes from the sigma-model perspective. They explicitly verified nilpotency of the charge matrix for certain supersymmetric fivedimensional black holes. However, a detailed study of various nilpotent orbits and how they relate to the known phase diagrams of five-dimensional black holes in a supergravity theory has been largely missing in the literature. The primary purpose of this paper is to fill this gap in the special case of minimal supergravity.

The main motivation for this study is to develop algebraic tools that can let us find new black hole solutions of physical interest. Many such solutions are still not known, for example, a black ring that describes thermal excitations above the supersymmetric black ring is not known (it was conjectured to exist in [29]; see [20] for a more detailed discussion), and certain dyonic extremal black rings, conjectured to exist in $[17,20]$, are also not known. Another motivation is to understand how different nilpotent orbits, and their partial orderings, are to be interpreted from the five-dimensional spacetime point of view. We also hope that such a study will give us further insights into the attractor mechanism for five-dimensional extremal black holes.

The rest of the paper is organized as follows. In section 2 we review salient features of the CvetičYoum family of metrics [31] and discuss its various extremal limits. In particular, we discuss two classes of regular cohomogeneity one extremal black holes contained in this family. In section 3 we present a general discussion of five-dimensional spacetimes with two commuting Killing vectors from the threedimensional sigma-model perspective. We show that the asymptotic boundary conditions relevant for five-dimensional black holes naturally give rise to a reductive decomposition of the Lie algebra of the hidden symmetry group of the theory. We then use this decomposition to relate five-dimensional spacetime charges to the Lie algebra valued Noether charge matrix of the sigma-model. In section 4 we show that the nilpotent orbits of $\mathrm{G}_{2(2)}$ are in one-to-one correspondence with phases of regular extremal cohomogeneity one black holes of minimal supergravity obtained as limits of the Cvetič-Youm solution. We close with a brief discussion in section 5. Technical details are regelated to appendices. In appendix A we recall the Cvetič-Youm solution. In appendix B we explicitly present the reductive decomposition of $\mathfrak{g}_{2(2)}$ relevant for the five-dimensional asymptotically flat boundary conditions. We remark that this work is largely a continuation of [17]. For a thorough discussion of nilpotent orbits of $\mathrm{G}_{2(2)}$ we refer the reader to [17].

\section{The Cvetič-Youm family and its extremal limits}

In this section we review certain properties of the Cvetič-Youm family of metrics. These metrics are studied in detail in $[30,31,32,33,34]$ so we shall be brief. Our main interest is in its various extremal limits and their sigma-model description.

\subsection{Preliminaries}

The theory we are interested in is minimal ungauged supergravity in five dimensions. It contains a metric $g_{5}$ and a gauge potential $A$ whose field strength is $F=d A$. The bosonic part of the Lagrangian 
takes the form of Einstein-Maxwell theory with a Chern-Simons term,

$$
\mathcal{L}_{5}=R_{5} \star_{5} \mathbf{1}-\frac{1}{2} \star_{5} F \wedge F+\frac{1}{3 \sqrt{3}} F \wedge F \wedge A .
$$

Upon dimensional reduction on a circle this theory becomes the so called $N=2, D=4$, $\mathrm{S}^{3}$ supergravity, which was analyzed from a group theory perspective in detail in [17]. Four-dimensional asymptotically flat black holes of the $S^{3}$ supergravity when uplifted to minimal supergravity describe asymptotically Kaluza-Klein black holes in five dimensions. In this paper we are interested in five-dimensional asymptotically flat boundary conditions as opposed to the Kaluza-Klein boundary conditions explored in [17].

We parameterize five-dimensional Minkowski metric as

$$
d s_{5}^{2}\left(\mathbb{R}^{4,1}\right)=-d t^{2}+d r^{2}+\frac{r^{2}}{4}\left(d \theta^{2}+d \phi^{2}+d \psi^{2}+2 \cos \theta d \psi d \phi\right),
$$

where $r$ is the radial coordinate and $\theta, \phi$, and $\psi$ are the standard Euler angles on the three-sphere with

$$
0 \leq \theta<\pi, \quad 0 \leq \phi<2 \pi, \quad 0 \leq \psi<4 \pi .
$$

The action of $\partial_{\psi}$ and $\partial_{\phi}$ as Killing fields commute, but they are not orthogonal as vectors in general. In this paper we exclusively work with metrics with three commuting Killing symmetries generating an $\mathbb{R} \times \mathrm{U}(1) \times \mathrm{U}(1)$ isometry group. However, to have a three-dimensional picture of black holes as geodesics on a coset manifold, this isometry group is not sufficiently large. One must look at metrics where one of the U(1)'s is enhanced to an SU(2) symmetry. This happens for a class of cohomogeneity one metrics. This class includes, for example, the supersymmetric BMPV black hole [35].

From the assumption of stationarity, we can define the mass as the Komar mass by integrating a two-form constructed from the time-like Killing vector $\partial_{t}$ over the three sphere at infinity as

$$
M=\frac{3}{32 \pi G_{5}} \int_{S_{\infty}^{3}} \star_{5} K
$$

where $K=d g$, and $g=g_{t \nu} d x^{\nu}$, and $G_{5}$ is Newton's constant in five dimensions. Similarly, from the Killing vectors $\partial_{\psi}$ and $\partial_{\phi}$ generating the rotation in the $\psi$ and $\phi$-planes respectively, we define the angular momentum by a similar Komar integral as

$$
J=\frac{1}{16 \pi G_{5}} \int_{S_{\infty}^{3}} \star_{5} K^{\prime}
$$

where now $K^{\prime}=d g^{\prime}$, and $g^{\prime}=g_{\mu \psi} d x^{\mu}$ or $g^{\prime}=g_{\mu \phi} d x^{\mu}$. The electric charge we define as

$$
Q_{\mathrm{E}}=\frac{1}{16 \pi G_{5}} \int_{S_{\infty}^{3}}\left(\star_{5} F-\frac{1}{\sqrt{3}} F \wedge A\right) .
$$

In our conventions, the BPS bound becomes

$$
M \geq \sqrt{3}\left|Q_{\mathrm{E}}\right|
$$

For completeness, let us also briefly recall the sigma-model machinery that we will use later in the paper. The Lagrangian (2.1) of minimal supergravity when reduced over the orbits of two commuting 
Killing vectors $\partial_{t}$ and, say, $\partial_{\psi}$ gives rise to three dimensional Euclidean gravity on a manifold $\mathcal{M}_{3}$ coupled to a sigma-model of maps from $\mathcal{M}_{3}$ to the target space $\mathrm{G}_{2(2)} / \mathrm{SO}_{0}(2,2)$. This sigma-model was derived and analysed in $[22,10]$. In this work we follow the conventions of $[10,17]$. To arrive at the three dimensional theory we use the reduction ansatz

$$
\begin{aligned}
\mathrm{d} s_{5}^{2} & =e^{\frac{1}{\sqrt{3}} \phi_{1}+\phi_{2}} d s_{3}^{2}-e^{\frac{1}{\sqrt{3}} \phi_{1}-\phi_{2}}\left(d t+\omega_{3}\right)^{2}+e^{-\frac{2}{\sqrt{3}} \phi_{1}}\left(d \psi+B_{1}+\chi_{1} d t\right)^{2}, \\
A & =B_{2}+\chi_{3} d t+\chi_{2} d \psi .
\end{aligned}
$$

In terms of the fields introduced through this ansatz we can rewrite the formulae for mass (2.4), angular momentum (2.5), and charge (2.6) as

$$
M=\frac{3 \pi}{16 G_{5}} \lim _{r \rightarrow \infty}\left(r^{3} \partial_{r} e^{\frac{1}{\sqrt{3}} \phi_{1}-\phi_{2}}\right), \quad J_{\psi}=\frac{\pi}{8 G_{5}} \lim _{r \rightarrow \infty}\left(r^{3} \partial_{r}\left(e^{-\frac{2}{\sqrt{3}} \phi_{1}} \chi_{1}\right)\right), \quad Q_{\mathrm{E}}=\frac{\pi}{8 G_{5}} \lim _{r \rightarrow \infty}\left(r^{3} \partial_{r} \chi_{3}\right),
$$

where we have implicitly assumed the standard $1 / r^{2}$ fall off for various metric and vector field components in asymptotically cartesian coordinates. In practice this simply means that the falloff of the scalars are such that limits (2.10) converge.

\subsection{Cvetič-Youm, BMPV, and extremal non-supersymmetric black holes}

A four parameter family of charged rotating black holes of five-dimensional minimal supergravity was obtained by Cvetič and Youm in [31] by applying boosts and string dualities to the (neutral, rotating) Myers-Perry black hole in five dimensions. The four parameters specifying the solution can be chosen to be the mass $M$, two angular momenta $J_{\phi}, J_{\psi}$ and electric charge $Q_{\mathrm{E}}$. It is convenient to present the solution in terms of the parameters $m, l_{1}, l_{2}$, and $\delta$. We recall the full solution in appendix A.

We restrict ourselves to the cohomogeneity one subclass of this solution, which is obtained by setting $l_{1}=l_{2}$ in the form of the metric presented in appendix $\mathrm{A}$ and changing from the Hopf coordinates $\left(\bar{\theta}, \phi_{1}, \phi_{2}\right)$ on the three-sphere to the Euler angular coordinates $(\theta, \phi, \psi)$ introduced above. Setting $l_{1}=l_{2}=: l$ enhances the isometry group from $\mathbb{R} \times \mathrm{U}(1) \times \mathrm{U}(1)$ to $\mathbb{R} \times \mathrm{SU}(2) \times \mathrm{U}(1)$. This enhancement of the rotational symmetry allows us to describe the solution as a geodesic on the coset manifold. In terms of the parameters $m, l$, and $c=\cosh \delta, s=\sinh \delta$ the physical charges (2.10) are

$$
M=\frac{3 \pi}{4 G_{5}} m\left(1+2 s^{2}\right), \quad J_{\psi}=\frac{\pi}{4 G_{5}} m l\left(c^{3}+s^{3}\right), \quad J_{\phi}=0, \quad Q_{\mathrm{E}}=\frac{\sqrt{3} \pi}{2 G_{5}} m c s .
$$

Writing the metric in the canonical form (2.8) we also observe that the one-form $\omega_{3}$ in (2.8) vanishes identically.

There are two regular extremal limits of this subclass of the Cvetič-Youm family. One of these limits is obtained by taking $m \rightarrow 0, l \rightarrow 0, \delta \rightarrow \infty$ keeping the mass, angular momentum and charge finite. In this limit we recover the supersymmetric BMPV black hole. The entropy of the BMPV black hole is given as

$$
S_{\mathrm{BMPV}}=2 \pi \sqrt{N_{\mathrm{M} 2}^{3}-4 J_{\psi}^{2}},
$$

where $N_{\mathrm{M} 2}=\frac{1}{\sqrt{3}} \ell_{p} Q_{\mathrm{E}}$ is the number of M2 branes, and $\ell_{p}$ is the five-dimensional planck length $\ell_{p}=$ $\left(\frac{4 G_{5}}{\pi}\right)^{1 / 3}$. On the BMPV branch angular momentum $J_{\psi}$ can be continuously taken to zero from a finite 
non-zero value while maintaining regularity of the metric. In the limit $J_{\psi}=0$ the solution simply reduces to the supersymmetric 5D Reissner-Nordstöm black hole. On this branch $Q_{\mathrm{E}}^{3}$ must be greater than $4 J_{\psi}^{2}$, as otherwise the solution develops naked closed timelike curves. The causal structure of the 'over-rotating' BMPV spacetime has been discussed in thorough detail in [36].

The second extremal limit is obtained by taking $m=2 l^{2}$. This produces an extremal but nonsupersymmetric black hole. Since the black hole is non-supersymmetric it does not saturates the BPS bound as can be easily checked from the expressions (2.11). The entropy of the black holes on this branch is given as

$$
S_{\text {extremal }}=2 \pi \sqrt{4 J_{\psi}^{2}-N_{\mathrm{M} 2}^{3}} .
$$

In this family the electric charge $Q_{\mathrm{E}}$ can be continuously taken to zero from a finite non-zero value while maintaining regularity of the metric. In the limit $Q_{\mathrm{E}}=0$ the solution reduces to extremal equally rotating Myers-Perry black hole. On this branch $N_{\mathrm{M} 2}^{3}$ must be less than $4 J_{\psi}^{2}$, otherwise the solution develops naked closed timelike curves.

\section{Describing five dimensional black holes as geodesics}

In this section we discuss how to describe five-dimensional asymptotically flat black holes as geodesics. Let $\mathcal{V}: \mathcal{M}_{3} \rightarrow \mathrm{G}_{2(2)} / \mathrm{SO}_{0}(2,2)$ be the coset map. If $\mathcal{V}$ only depend on the radial coordinate $r$ on $\mathcal{M}_{3}$ then the equations of motion for the sigma-model imply that $\mathcal{V}$ should trace out a geodesic on the coset manifold [37]. Define $\phi(g)=\left(g^{T} \eta_{4}\right)^{-1} \eta_{4}$ where $\eta_{4}$ is the symmetric matrix preserved by $\mathrm{SO}(2,2)$. We have that $\phi(g)=g$ if $g \in \mathrm{SO}(2,2)$. Using the map $\phi$ we define the group element

$$
M=\phi(\mathcal{V})^{-1} \mathcal{V}
$$

A solution to the equations of motion is now given by

$$
M=M_{0} \exp (\tau \mathcal{Q})
$$

provided $\phi(M)=M^{-1}$. Assuming the base space $\mathcal{M}_{3}$ to be flat,

$$
d s^{2}=\frac{r^{2}}{4}\left[d r^{2}+\frac{1}{4} r^{2} d \theta^{2}+\frac{1}{4} r^{2} \sin ^{2} \theta d \phi^{2}\right],
$$

Einstein's equations allow us to relate the affine parameter $\tau$ to the radial coordinate $r$. For an extremal five-dimensional black hole this gives $\tau=-\frac{4}{r^{2}}$. We interpret (3.2) as giving all geodesics from the point $M_{0}$. To describe asymptotically flat black holes we need to find the point $M_{0}$ from where the corresponding geodesics start, and solve the constraint $\phi(M)=M^{-1}$ at this point. This translates to finding a reductive decomposition

$$
\mathfrak{g}_{2(2)}=\mathfrak{k}_{5} \oplus \mathfrak{p}_{5}
$$

of the Lie algebra $\mathfrak{g}_{2(2)}$ such that $\mathfrak{p}_{5} \cong T_{M_{0}}(G / \tilde{K})$ and $\mathfrak{k}_{5}$ is the Lie algebra of the group $K_{5} \cong \mathrm{SO}_{0}(2,2)$ preserving the point $M_{0}$.

To answer these questions, let us now recall how the similar construction work for asymptotically flat black holes in four dimensions. Assuming that the scalar fields in the sigma-model vanish at infinity, 
we have $M_{0}=I$, where $I$ is the identity element in $\mathrm{G}_{2(2)}$. This implies that $\mathcal{Q} \in \mathfrak{g}_{2(2)}$ in (3.2) should obey

$$
\mathcal{Q}^{T} \eta_{4}-\eta_{4} \mathcal{Q}=0
$$

Using $\eta_{4}$ we get the reductive decomposition $\mathfrak{g}_{2(2)}=\mathfrak{k}_{4} \oplus \mathfrak{p}_{4}$, where $\mathfrak{k}_{4}=\mathfrak{s o}(2,2)$ is the Lie algebra of the $\mathrm{SO}(2,2)$ group preserving the identity and $\mathfrak{p}_{4}$ is defined as the space of elements obeying (3.5), so that $T_{I}(G / \tilde{K}) \cong \mathfrak{p}_{4}$. Let us now investigate how all this translate to five-dimensional asymptotically flat black holes. See also [21].

To derive the asymptotic initial point $M_{0}$ we reduce five-dimensional Minkowski space (2.2) to our $\mathrm{G}_{2(2)}$ sigma model. From the reduction ansatz (2.8) we find

$$
\phi_{1}=-\frac{\sqrt{3}}{2} \log \frac{r^{2}}{4}, \quad \phi_{2}=-\frac{1}{2} \log \frac{r^{2}}{4}, \quad \chi_{5}=\frac{r^{2}}{4},
$$

and all other fields to be zero. Here $\chi_{5}$ is the scalar dual to $B_{1}$. This implies two things: (i) that five-dimensional Minkowski space is in fact described by a non-trivial geodesic on the coset manifold, contrary to the four dimensional case, and (ii) that the scalars diverge at infinity. However (3.1) constructed from the coset representative $\mathcal{V}_{\text {Mink }}$ take a finite value when $r \rightarrow \infty$. Thus, we find, in the notation of [10], that

$$
M_{0} \equiv \lim _{r \rightarrow \infty} M_{\text {Mink }}=\exp \left(-\frac{\pi}{12}\left(E_{5}-F_{5}\right)\right),
$$

where $E_{5}$ and $F_{5}$ are Chevalley-Serre generators of $\mathfrak{g}_{2(2)}$.

Having found the point $M_{0}$ we can define a metric $\eta_{5}:=\eta_{4} M_{0}$ such that $K_{5}$ is the Lie subgroup of $\mathrm{G}_{2(2)}$ that preserves this metric. It is easy to see that for $g \in \mathrm{G}_{2(2)}$ with $g^{T} \eta_{5} g=\eta_{5}$ and if the coset representative transform as $\mathcal{V} \rightarrow \mathcal{V} g$, then the asymptotic point $M_{0}$ is preserved. Furthermore, if $M$ is given by (3.2), then the right action $\mathcal{V} \rightarrow \mathcal{V} g$, imply that $\mathcal{Q} \rightarrow A d_{g} \mathcal{Q}$. So, the reductive decomposition (3.4) is defined by elements satisfying $\mathcal{Q}^{T} \eta_{5} \pm \eta_{5} \mathcal{Q}=0$, where we have + for elements in $\mathfrak{k}_{5}$ and - for elements in $\mathfrak{p}_{5}$, just as for the four dimensional decomposition. We conclude that asymptotically flat black holes map to elements $\mathcal{Q} \in \mathfrak{p}_{5}$, and under the action of the group preserving the asymptotics, these elements transform by conjugation. Exactly as in the four dimensional case we can hence classify different types of black hole solutions by the orbits of the group $K_{5}$. The space $\mathfrak{p}_{5}$ is eight-dimensional and spanned by the elements $Y_{i}, i=1, \ldots, 8$. Details about $Y_{i}$ 's and the elements spanning $\mathfrak{k}_{5}$ are given in Appendix B. The eight elements $Y_{i}$ correspond to 'conserved charges' (or more correctly to 'conserved physical properties') of the black hole simply by the geodesic property. We now find the mapping between the eight elements of $\mathfrak{p}_{5}$ and physical properties of the five-dimensional spacetimes. These issues are also discussed in [28]. See also [21].

We assume $\mathcal{Q}^{3}=0$ and work with the three-dimensional base metric (3.3). From the general nilpotent charge matrix $\mathcal{Q}$ we construct the asymptotic spacetime fields and find the map from conserved quantities in the sigma-model to the conserved quantities in spacetime. With a lot of hind-sight we parameterize the tangent vectors $\mathcal{Q}$ in $\mathfrak{p}_{5}$ as

$$
\mathcal{Q}=-N Y_{1}+\beta_{1} Y_{2}+\frac{1}{\pi} G_{5} Q_{\mathrm{E}} Y_{3}-\frac{2}{\pi} G_{5} J_{\psi} Y_{4}+\frac{2 \sqrt{3}}{\pi} G_{5} J_{\mathrm{M}} Y_{5}+\frac{1}{2 \pi \sqrt{3}} G_{5} M Y_{6}+\beta_{2} Y_{7}+p_{0} Y_{8}
$$


Normalization of various coefficients here is chosen for later convenience. We now expand the fields, one at the time, in the reduction ansatz (2.8) for large values of the radial coordinate $r$. The $\phi$-component of the one form $\omega_{3}$ expands as

$$
\left.\omega_{3}\right|_{\phi} \simeq N \cos \theta+\mathcal{O}\left(\frac{1}{r^{2}}\right)
$$

This changes asymptotics of the spacetime. Therefore for asymptotic flatness we demand $N=0$. Expanding the three scalar fields $\chi_{1}, \chi_{2}$ and $\chi_{3}$ we find

$$
\begin{aligned}
& \chi_{1} \simeq \frac{4 N}{r^{2}}+\mathcal{O}\left(\frac{1}{r^{4}}\right), \quad \chi_{2} \simeq \frac{\beta_{1}}{p_{0}}+\mathcal{O}\left(\frac{1}{r^{2}}\right), \quad \text { and } \\
& \chi_{3} \simeq \frac{1}{r^{2}}\left(-\frac{4 \beta_{1}^{2}}{\sqrt{3} p_{0}}+\frac{4 N \beta_{1}}{p_{0}}-\frac{4 G_{5} Q_{\mathrm{E}}}{\pi}\right)+\mathcal{O}\left(\frac{1}{r^{4}}\right) .
\end{aligned}
$$

Note that parameter $\beta_{1}$ changes the asymptotic form of $\chi_{3}$; however, since it enters as a constant term in the expansion of $\chi_{2}$ it can be gauged away under certain natural assumptions on the nature of the spacetime. In the examples we consider $\beta_{1}$ always turns out to be zero. The $\phi$-component of the one form $B_{1}$ goes as

$$
\left.B_{1}\right|_{\phi} \simeq p_{0} \cos \theta+\mathcal{O}\left(\frac{1}{r^{2}}\right) .
$$

The parameter $p_{0}$ is thus the Chern class of the $\psi$-circle over the two-sphere parameterized by $(\theta, \phi)$. Therefore, $p_{0}$ is naturally interpreted as the orbifolding parameter, leading to constant $t, r$ hypersurfaces to be $S^{3} / \mathbb{Z}_{p_{0}}$ for large values of $r$ [28]. For asymptotic flatness we demand $p_{0}=1$. Let us therefore put $N=\beta_{1}=0$ and $p_{0}=1$ in the rest of the paper. The $\phi$-component of the one-form $B_{2}$ then expands as

$$
\left.B_{2}\right|_{\phi} \simeq \frac{4 \sqrt{3} G_{5} J_{\mathrm{M}} \cos \theta}{\pi r^{2}}+\mathcal{O}\left(\frac{1}{r^{4}}\right)
$$

This term is like a 'dipolar magnetic flux' through the three-sphere. We will see later that for rotating electrically charged black holes this term is non-zero. Intuitively, it captures the angular momentum contained in the Maxwell field; for this reason we denote it by $J_{\mathrm{M}}$. Finally, let us consider the two dilatons by expanding the $g_{t t}$ and $g_{t \psi}$ components. We find

$$
g_{t t} \simeq-1+\frac{8 G_{5} M}{3 \pi r^{2}}+\mathcal{O}\left(\frac{1}{r^{4}}\right), \quad g_{t \psi} \simeq-\frac{4 G_{5} J_{\psi}}{\pi r^{2}}+\mathcal{O}\left(\frac{1}{r^{4}}\right) .
$$

We conclude that $M$ refers to the mass, $Q_{\mathrm{E}}$ to the electric charge and $J_{\psi}$ to the angular momentum. Note that $\beta_{2}$ has so far not made an appearance. It can be thought of as an auxiliary sigma-model charge.

Although, in the above analysis we have assumed $\mathcal{Q}^{3}=0$ and have only worked with the threedimensional base metric (3.3), we expect the analysis to hold more generally. In particular, we will see in the next section that it applies to non-extremal black holes as well.

\section{Nilpotent orbits and phases of extremal black holes}

In this section we consider nilpotent $\mathrm{SO}_{0}(2,2)$-orbits in $\mathfrak{p}_{5}$ and show that the partial ordering of nilpotent orbits of $\mathrm{G}_{2(2)}$ is in one-to-one correspondence with the phase diagram of extremal limits of the 
cohomogeneity one Cvetič-Youm black hole. The charge matrix of the non-extremal Cvetič-Youm black hole can be readily calculated, and it is given by

$$
\mathcal{Q}=\frac{\sqrt{3}}{2} m c s Y_{3}-\frac{1}{2} m l\left(c^{3}+s^{3}\right) Y_{4}+\frac{\sqrt{3}}{2} m l c s(c+s) Y_{5}+\frac{\sqrt{3}}{8} m\left(1+2 s^{2}\right) Y_{6}+\frac{1}{16} m\left(m-8 l^{2}\right) Y_{7}+Y_{8} .
$$

Note that the identification of charges derived in section 3 matches with the charges given in section 2 . In particular $N=\beta_{1}=0$ and $p_{0}=1$. It can be easily verified that the charge matrix $\mathcal{Q} \in \mathfrak{p}_{5}$ obey the characteristic equation of [9], namely

$$
\mathcal{Q}^{3}-\frac{1}{4} \operatorname{tr}\left(\mathcal{Q}^{2}\right) \mathcal{Q}=0
$$

with

$$
\operatorname{tr}\left(\mathcal{Q}^{2}\right)=m\left(m-2 l^{2}\right) .
$$

Note that the right hand side of this equation is independent of the electric charge parameter $\delta$. This is a reflection of the fact that the Cvetič-Youm black hole lies in the $\mathrm{SO}_{0}(2,2)$ orbit of the Myers-Perry black hole $[22,10]$. In fact, Cvetič and Youm constructed their solution by applying boosts and string dualities which is equivalent to applying certain $\mathrm{SO}_{0}(2,2)$ transformations on the Myers-Perry black hole. The extremal limits $\operatorname{tr}\left(\mathcal{Q}^{2}\right) \rightarrow 0$ then give the nilpotent orbits of interest, as the zeros of equation (4.3) are exactly the two cases we discussed in section 2. We now analyze these limits in more detail: we first recall the discussion of nilpotent orbits from [17] and then look at the two branches.

The nilpotent orbits for the three dimensional sigma-model of minimal five-dimensional supergravity were analyzed in detail in [17]. The focus there was on asymptotically Kaluza-Klein black holes, however, all of the group theoretical analysis carries over to the present setting without any modification. Following [38], reference [17] gave a detailed construction of representatives of all $\mathrm{SO}_{0}(2,2)$ orbits. Via the reductive decomposition (3.4) described in the previous section, we can immediately read off representatives for the nilpotent orbits of interest. We will not repeat the details of [17] here; we simply remind the reader of the most important features. From [38] we know that nilpotent elements of $\mathfrak{g}_{2(2)}$ fit in five orbits $\mathcal{O}_{i}, i=1, \ldots, 5$ under the adjoint action of $\mathrm{G}_{2(2)}$. These five orbits satisfy a partial ordering such that $\mathcal{O}_{i}$ is in the closure (in the usual topological sense) of some $\mathcal{O}_{j}$ with $i<j$. In figure 1 a Hasse diagram of the five nilpotent orbits of $\mathrm{G}_{2(2)}$ is reproduced from [39]. The Hasse diagram encodes the fact $\mathcal{O}_{3}$ and $\mathcal{O}_{4}$ are in the closure of $\mathcal{O}_{5}$, and similarly that $\mathcal{O}_{1}$ is in the closure of $\mathcal{O}_{2}$, which in turn is in the closure of both $\mathcal{O}_{3}$ and $\mathcal{O}_{4}$. When restricting to $\mathrm{SO}_{0}(2,2)$ orbits of $\mathfrak{p}_{4}$ (or $\mathfrak{p}_{5}$ ), there is a possibility for these orbits to split into several $\mathrm{SO}_{0}(2,2)$ orbits. This happens for $\mathcal{O}_{3}$ and $\mathcal{O}_{4}$ [17]. Both $\mathcal{O}_{3}$ and $\mathcal{O}_{4}$ 'split' into two smaller orbits each in $\mathfrak{p}_{4}$ (or $\left.\mathfrak{p}_{5}\right)$. A similar splitting does not happen for $\mathcal{O}_{1}$ and $\mathcal{O}_{2}$. The $\mathcal{O}_{5}$ orbit has not been analyzed in detail in the literature yet. The details of the $\mathcal{O}_{5}$ orbit does not concern us here, as the nilpotency degree of this orbit is too high to arise as the extremal limit of the Cvetič-Youm black holes. The four $\mathrm{SO}_{0}(2,2)$ orbits for $\mathcal{O}_{3}$ and $\mathcal{O}_{4}$ are denoted [17] $\mathcal{O}_{3 K}, \mathcal{O}_{3 K}^{\prime}, \mathcal{O}_{4 K}$ and $\mathcal{O}_{4 K}^{\prime}$. It was shown in [17], confirming the conjecture in [15], that only one 'splitted' orbit in each $\mathrm{G}_{2(2)}$ orbit corresponded to physical black holes; the others having singularities outside the horizon. We now show that a similar story holds for the five-dimensional asymptotically flat setting in minimal supergravity. It is natural to conjecture, following [15], that a similar story holds for all five-dimensional theories. 


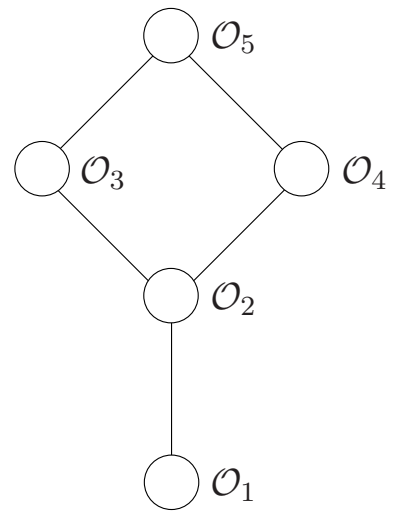

Figure 1: Hasse diagram for the partial ordering of the nilpotent orbits in $\mathfrak{g}_{2(2)}$.

Instead of following the method of [17], where certain representatives of the orbits were used to generate the full orbit space, it turns out that we can solve the $\mathcal{Q}^{3}=0$ equation explicitly. This difference arises because in the analysis of [17] the only simplification made was setting the four dimensional NUT charge to zero, which is not enough for the equation $\mathcal{Q}^{3}=0$ to be solved explicitly, and as a result it was much more practical to generate orbits using the adjoint action of $\mathrm{G}_{2(2)}$ on orbit representatives. In this paper we set $\beta_{1}=0, N=0$ and $p_{0}=1$. This simplifies the problem considerably. In fact, in the parametrization (3.7) of $\mathfrak{p}_{5}$, we find that the solution space of the nilpotency equation $\mathcal{Q}^{3}=0$ has two distinct branches. We now turn to the study of these branches.

\subsection{Supersymmetric branch}

The first branch is

$$
\beta_{2}=0, \quad J_{\mathrm{M}}=J_{\psi}, \quad M=\sqrt{3} Q_{\mathrm{E}} .
$$

We immediately see that this solution saturates the five-dimensional BPS bound. In fact the solution is supersymmetric. The condition that the mass be positive translates to the condition that electric charge must be positive. The space-time solution derived from the geodesic is exactly the BMPV solution. Thus, as expected, (4.4) reproduces the $m \rightarrow 0, l \rightarrow 0, \delta \rightarrow \infty$ limit of the Cvetič-Youm black hole. As is well known, for the BMPV solution to have all closed time-like curves hidden behind the horizon, we need

$$
N_{\mathrm{M} 2}^{3}>4 J_{\psi}^{2} .
$$

In our way of seeing it, this is equivalent to the condition that the exponentials in (2.8) are always positive. In fact, for BMPV we have

$$
\lim _{r \rightarrow 0} e^{-\frac{2}{\sqrt{3}} \phi_{1}}=\left(\frac{G_{5}}{2 \pi}\right)^{2 / 3} \frac{N_{\mathrm{M} 2}^{3}-4 J_{\psi}^{2}}{N_{\mathrm{M} 2}^{2}},
$$

so for the exponentials to be well-defined we need to satisfy (4.5). Group theoretically this bound is equivalent to the border between the two $\mathrm{SO}_{0}(2,2)$ orbits $\mathcal{O}_{3 K}$ and $\mathcal{O}_{4 K}$. $\mathcal{O}_{4 K}$ contain the over-rotating BMPV spacetime and $\mathcal{O}_{3 K}$ the under-rotating. That (4.5) is violated also signals closed time-like curves 


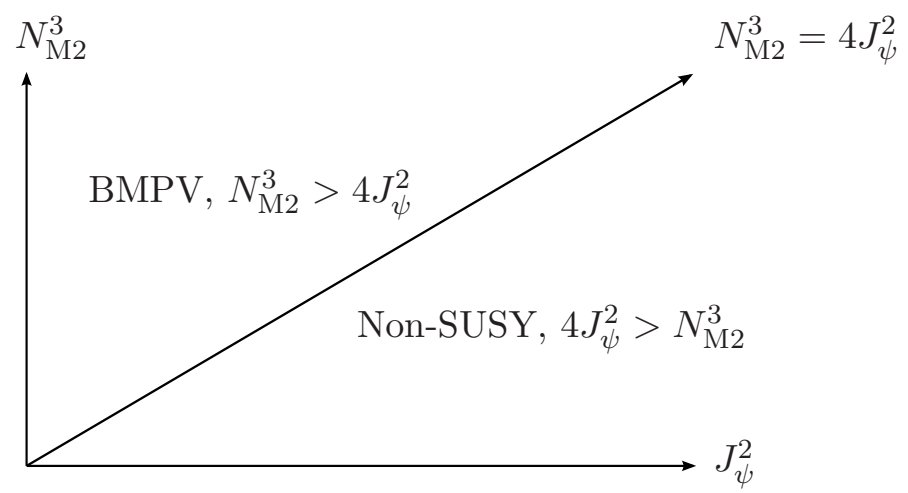

Figure 2: A phase diagram of the extremal limits of the cohomogeneity one Cvetič-Youm black hole. The line $N_{\mathrm{M} 2}^{3}=4 J_{\psi}^{2}$ corresponds to the $\mathcal{O}_{2}$ orbit, the region above the line corresponds to the $\mathcal{O}_{3 K}$ orbit and the region below the line corresponds to $\mathcal{O}_{4 K}^{\prime}$ orbit. The origin being Minkowski space corresponds to $\mathcal{O}_{1}$. Compare this phase diagram with the Hasse diagram in figure 1.

outside of the horizon, since (4.6) is the $g_{\psi \psi}$-component of the metric for the black hole. See [36] for a more detailed discussion about this point. One also sees that the entropy

$$
S=2 \pi \sqrt{N_{\mathrm{M} 2}^{3}-4 J_{\psi}^{2}},
$$

is ill-defined unless we obey (4.5). When the bound (4.5) is saturated we are in the orbit $\mathcal{O}_{2}$, a zerohorizon black hole with $4 J_{\psi}^{2}=N_{\mathrm{M} 2}^{3}$. Furthermore, if all charges vanish we end up in the $\mathcal{O}_{1}$ orbit, where the only solution is Minkowski space.

\subsection{Non-supersymmetric branch}

The other branch is given by

$$
M=\frac{18 \pi}{G_{5}} \frac{J_{\mathrm{M}}^{2}}{Q_{\mathrm{E}}^{2}}-\sqrt{3} Q_{\mathrm{E}}, \quad J_{\psi}=\frac{12 \sqrt{3} \pi}{G_{5}} \frac{J_{\mathrm{M}}^{3}}{Q_{\mathrm{E}}^{3}}-3 J_{\mathrm{M}}, \quad \beta_{2}=\frac{12 \sqrt{3} G_{5}}{\pi} \frac{J_{\mathrm{M}}^{2}}{Q_{\mathrm{E}}}-108 \frac{J_{\mathrm{M}}^{4}}{Q_{\mathrm{E}}^{4}} .
$$

Here, to satisfy the BPS bound, we have to obey the condition

$$
4 J_{\psi}^{2}>N_{\mathrm{M} 2}^{3}
$$

This branch corresponds to the $m=2 l^{2}$ extremal limit of the cohomogeneity one Cvetič-Youm black hole. If we consider the positivity of the exponentials in the solution, we find that the condition (4.9) is sufficient for the metric to be well-defined. Exactly opposite to the supersymmetric branches above, the physical orbit $\mathcal{O}_{4 K}^{\prime}$ now contain the over-rotating black hole, and the unphysical orbit $\mathcal{O}_{3 K}^{\prime}$ contain the under-rotating spacetime. The under-rotating spacetime violates the BPS-bound. The regular solution on this branch is analyzed in detail in [34]. We note that $g_{\psi \psi}$ becomes negative at finite positive $r$ if $4 J_{\psi}^{2}<N_{\mathrm{M} 2}^{3}$, making closed orbits of $\partial_{\psi}$ time-like. In figure 2 we illustrate the phase space of physical solutions in terms of the charges $N_{\mathrm{M} 2}$ and $J_{\psi}$. Note the similarities between this phase diagram and the Hasse diagram of the partial ordering in figure 1. 


\section{Discussion}

In this paper we studied extremal cohomogeneity one five-dimensional asymptotically flat black holes of minimal supergravity in terms of the geodesics generated by nilpotent elements of the Lie algebra $\mathfrak{g}_{2(2)}$ on the coset manifold $\mathrm{G}_{2(2)} / \mathrm{SO}(2,2)$. We discussed two branches of regular extremal black holes with these properties: (i) the supersymmetric BMPV branch, and (ii) the non-supersymmetric extremal branch. We showed that these branches are reproduced by $\mathcal{O}_{3 K}$ and $\mathcal{O}_{4 K}^{\prime}$ nilpotent $\mathrm{SO}(2,2)$-orbits respectively. We also showed that the partial ordering of nilpotent orbits of $\mathrm{G}_{2(2)}$ is in one-to-one correspondence with the phase diagram of these extremal black holes.

The correspondence between phase diagrams of extremal four and five-dimensional black holes and the partial ordering of nilpotent orbits of the corresponding three-dimensional hidden symmetry group seems to be a generic phenomenon. So far this has only been explored for cohomogeneity one metrics. It is likely that these considerations extend beyond this assumption. For example extremal rotating Kaluza-Klein black holes (D0-D6) of vacuum five-dimensional gravity come in two branches: (i) slowly rotating branch, and (ii) fast rotating branch. The entropy for the slowly rotating solutions take the form

$$
S_{\text {slow }}=\pi \sqrt{N_{0}^{2} N_{6}^{2}-4 J^{2}}
$$

and for the fast rotating solutions take the form

$$
S_{\text {fast }}=\pi \sqrt{4 J^{2}-N_{0}^{2} N_{6}^{2}} .
$$

Given the similarity of these expressions with equations (2.12) and (2.13) it may be true that the consideration of nilpotent orbits extend to cohomogeneity two attractors as well. We hope to return to this question in the future.

\section{Acknowledgements}

We thank Boris Pioline for encouraging us to explore the five-dimensional asymptotically flat boundary conditions. We have benefited from our discussions with Guillaume Bossard, Stefano Giusto, and Clement Ruef. Our work is partially supported by IISN - Belgium (conventions 4.4511.06 and 4.4514.08) and by the Belgian Federal Science Policy Office through the Interuniversity Attraction Pole P6/11.

\section{A Cvetič-Youm metric}

For completeness we present the Cvetič-Youm solution in this appendix. See also [30, 31, 32, 33, 34, 40]. The metric and the vector potential take the form

$$
\begin{gathered}
d s^{2}=g_{t t} d t^{2}+2 g_{t \phi_{1}} d t d \phi_{1}+2 g_{t \phi_{2}} d t d \phi_{2}+g_{\phi_{1} \phi_{1}} d \phi_{1}^{2}+g_{\phi_{2} \phi_{2}} d \phi_{2}^{2}+2 g_{\phi_{1} \phi_{2}} d \phi_{1} d \phi_{2}+g_{r r} d r^{2}+g_{\bar{\theta} \bar{\theta}} d \bar{\theta}^{2} \\
A=A_{t} d t+A_{\phi_{1}} d \phi_{1}+A_{\phi_{2}} d \phi_{2}
\end{gathered}
$$


with

$$
\begin{aligned}
& g_{t t}=-\frac{\Sigma(\Sigma-2 m)}{\left(\Sigma+2 m s^{2}\right)^{2}} \\
& g_{t \phi_{1}}=-\frac{2 m \sin ^{2} \bar{\theta}\left[\Sigma\left\{l_{1} c^{3}+l_{2} s^{3}\right\}-2 m l_{2} s^{3}\right]}{\left(\Sigma+2 m s^{2}\right)^{2}} \text {, } \\
& g_{t \phi_{2}}=-\frac{2 m \cos ^{2} \bar{\theta}\left[\Sigma\left\{l_{2} c^{3}+l_{1} s^{3}\right\}-2 m l_{1} s^{3}\right]}{\left(\Sigma+2 m s^{2}\right)^{2}}, \\
& g_{\phi_{1} \phi_{1}}=\frac{\sin ^{2} \bar{\theta}}{\left(\Sigma+2 m s^{2}\right)^{2}}\left[\left(r^{2}+2 m s^{2}+l_{1}^{2}\right)\left(\Sigma+2 m s^{2}\right)^{2}\right. \\
& \left.+2 m \sin ^{2} \bar{\theta}\left\{\Sigma\left(l_{1}^{2} c^{2}-l_{2}^{2} s^{2}\right)-4 m l_{1} l_{2} c^{3} s^{3}-2 m s^{4}\left(l_{1}^{2} c^{2}+l_{2}^{2} s^{2}\right)-4 m l_{2}^{2} s^{4}\right\}\right], \\
& g_{\phi_{2} \phi_{2}}=\frac{\cos ^{2} \bar{\theta}}{\left(\Sigma+2 m s^{2}\right)^{2}}\left[\left(r^{2}+2 m s^{2}+l_{2}^{2}\right)\left(\Sigma+2 m s^{2}\right)^{2}\right. \\
& \left.+2 m \cos ^{2} \bar{\theta}\left\{\Sigma\left(l_{2}^{2} c^{2}-l_{1}^{2} s^{2}\right)-4 m l_{1} l_{2} c^{3} s^{3}-2 m s^{4}\left(l_{2}^{2} c^{2}+l_{1}^{2} s^{2}\right)-4 m l_{1}^{2} s^{4}\right\}\right] \text {, } \\
& g_{\phi_{1} \phi_{2}}=\frac{2 m \cos ^{2} \bar{\theta} \sin ^{2} \bar{\theta}\left[l_{1} l_{2}\left\{\Sigma-6 m s^{4}\right\}-2 m\left(l_{1}^{2}+l_{2}^{2}\right) s^{3} c^{3}-4 m l_{1} l_{2} s^{6}\right]}{\left(\Sigma+2 m s^{2}\right)^{2}} \\
& g_{r r}=\frac{r^{2}\left(\Sigma+2 m s^{2}\right)}{\left(r^{2}+l_{1}^{2}\right)\left(r^{2}+l_{2}^{2}\right)-2 m r^{2}}, \\
& g_{\bar{\theta} \bar{\theta}}=\Sigma+2 m s^{2} \text {, } \\
& A_{t}=-\frac{2 \sqrt{3} m s c}{\left(\Sigma+2 m s^{2}\right)}, \\
& A_{\phi_{1}}=-A_{t}\left(l_{1} c+l_{2} s\right) \sin ^{2} \bar{\theta}, \\
& A_{\phi_{2}}=-A_{t}\left(l_{2} c+l_{1} s\right) \cos ^{2} \bar{\theta} \text {. }
\end{aligned}
$$

For convenience we have defined

$$
\Sigma(r, \bar{\theta}) \equiv r^{2}+l_{1}^{2} \cos ^{2} \bar{\theta}+l_{2}^{2} \sin ^{2} \bar{\theta}
$$

Setting $\delta=0$ reproduces the five-dimensional MP black hole. In the above form Hopf coordinates are used on the $S^{3}$. In the main text we use Euler coordinates on the $S^{3}$. The two coordinate systems are related as

$$
\bar{\theta}=\frac{\theta}{2}, \quad \psi=\phi_{1}+\phi_{2}, \quad \phi=\phi_{2}-\phi_{1} .
$$

For calculational convenience we also use $x=\cos \bar{\theta}$. Our spacetime orientation is

$$
\epsilon_{r x \phi_{1} \phi_{2} t}=r \cos \bar{\theta}\left(\Sigma+2 m s^{2}\right) .
$$

\section{B Asymptotic reductive decomposition}

In this appendix we list the explicit form of $\mathfrak{k}_{5}$ and $\mathfrak{p}_{5}$ in the reductive decomposition

$$
\mathfrak{g}_{2(2)}=\mathfrak{k}_{5} \oplus \mathfrak{p}_{5}
$$


at the point $M_{0} \in \mathrm{G}_{2(2)} / \mathrm{SO}_{0}(2,2)$ derived in the main text. The subalgebra $\mathfrak{k}_{5}$ corresponding to $K_{5}$ is

$$
\mathfrak{k}_{5}=\operatorname{Span}_{\mathbb{R}}\left(X_{1}, X_{2}, X_{3}, X_{4}, X_{5}, X_{6}\right)
$$

with

$$
\begin{aligned}
& X_{1}=e_{1}-f_{6}, \quad X_{2}=f_{4}-f_{2}, \quad X_{3}=e_{3}+f_{3}, \\
& X_{4}=f_{1}-e_{6}, \quad X_{5}=e_{2}-e_{4}, \quad X_{6}=h_{1}+\frac{1}{\sqrt{3}} h_{2},
\end{aligned}
$$

where we follow the notation of [10] for the definitions of $h$ 's, $e$ 's, and $f$ 's. This subalgebra $\mathfrak{k}_{5}$ is isomorphic to $\mathfrak{s l}(2, \mathbb{R}) \oplus \mathfrak{s l}(2, \mathbb{R})$ as can be seen via the following change of basis

$$
\begin{aligned}
& \mathbf{h}_{1}=\frac{\sqrt{3}}{2}\left(X_{3}+\frac{3}{2} X_{6}\right), \quad \mathbf{e}_{1}=3\left(X_{4}-\frac{1}{\sqrt{3}} X_{5}\right), \quad \mathbf{f}_{1}=\frac{3}{8}\left(X_{1}+\frac{1}{\sqrt{3}} X_{2}\right), \\
& \mathbf{h}_{2}=-\frac{\sqrt{3}}{2}\left(X_{3}-\frac{1}{2} X_{6}\right), \quad \mathbf{e}_{2}=X_{4}+\sqrt{3} X_{5}, \quad \mathbf{f}_{2}=\frac{1}{8}\left(X_{1}-\sqrt{3} X_{2}\right) .
\end{aligned}
$$

The elements in $\mathfrak{g}_{2(2)}$ that span $\mathfrak{p}_{5}$ are

$$
\begin{aligned}
& Y_{1}=e_{1}+f_{6}, \quad Y_{2}=f_{4}+f_{2}, \quad Y_{3}=e_{3}-f_{3}, \quad Y_{4}=f_{1}+e_{6}, \\
& Y_{5}=e_{2}+e_{4}, \quad Y_{6}=h_{1}-\sqrt{3} h_{2}, \quad Y_{7}=e_{5}, \quad Y_{8}=f_{5} .
\end{aligned}
$$

These eight generators correspond to eight conserved charges in the three-dimensional sigma-model. Let us now diagonalize the adjoint action of $\mathbf{h}_{\mathbf{1}}$ and $\mathbf{h}_{\mathbf{2}}$ on $\mathfrak{p}_{5}$. By doing so we can immediately read of the representatives of nilpotent orbits from the discussion in [17]. Let $Y_{(m, n)}$ be the generators of $\mathfrak{p}_{5}$ with eigenvalues $m$ under $\mathbf{h}_{\mathbf{1}}$ and $n$ under $\mathbf{h}_{\mathbf{2}}$, then

$$
\begin{gathered}
Y_{(3,1)}=Y_{7}, \quad Y_{(1,1)}=Y_{4}+\frac{Y_{5}}{\sqrt{3}}, \quad Y_{(-1,1)}=Y_{3}-\frac{Y_{6}}{2}, \quad Y_{(-3,1)}=Y_{1}-\sqrt{3} Y_{2}, \\
Y_{(3,-1)}=Y_{4}-\sqrt{3} Y_{5}, \quad Y_{(1,-1)}=Y_{3}+\frac{Y_{6}}{2}, \quad Y_{(-1,-1)}=Y_{1}+\frac{Y_{2}}{\sqrt{3}}, \quad Y_{(-3,-1)}=Y_{8} .
\end{gathered}
$$

The truncation of the sigma-model to the pure gravity subalgebra $\mathfrak{s l}(3, \mathbb{R})$ is obtained by picking out the long roots. The isotropy subgroup for pure gravity $\mathfrak{s o}(2,1) \cong \mathfrak{s l}(2, \mathbb{R})$ is spanned by

$$
H=\sqrt{3} X_{6}, \quad E=2 X_{4}, \quad F=X_{1},
$$

and the coset space is spanned by $Y_{1}, Y_{4}, Y_{6}, Y_{7}$ and $Y_{8}$.

\section{References}

[1] A. Ceresole and G. Dall'Agata, "Flow Equations for Non-BPS Extremal Black Holes," JHEP 03 (2007) 110, arXiv:hep-th/0702088.

[2] L. Andrianopoli, R. D'Auria, E. Orazi, and M. Trigiante, "First Order Description of Black Holes in Moduli Space," JHEP 11 (2007) 032, arXiv:0706.0712 [hep-th]. 
[3] K. Hotta and T. Kubota, "Exact Solutions and the Attractor Mechanism in Non-BPS Black Holes," Prog. Theor. Phys. 118 (2007) 969-981, arXiv:0707.4554 [hep-th].

[4] D. Gaiotto, W. W. Li, and M. Padi, "Non-Supersymmetric Attractor Flow in Symmetric Spaces," JHEP 12 (2007) 093, arXiv:0710.1638 [hep-th].

[5] E. G. Gimon, F. Larsen, and J. Simon, "Black Holes in Supergravity: the non-BPS Branch," JHEP 01 (2008) 040, arXiv:0710.4967 [hep-th].

[6] E. Bergshoeff, W. Chemissany, A. Ploegh, M. Trigiante, and T. Van Riet, "Generating Geodesic Flows and Supergravity Solutions," Nucl. Phys. B812 (2009) 343-401,

arXiv:0806.2310 [hep-th].

[7] S. Bellucci, S. Ferrara, A. Marrani, and A. Yeranyan, "stu Black Holes Unveiled," arXiv:0807.3503 [hep-th].

[8] J. Perz, P. Smyth, T. Van Riet, and B. Vercnocke, "First-order flow equations for extremal and non-extremal black holes," JHEP 03 (2009) 150, arXiv:0810.1528 [hep-th].

[9] G. Bossard, H. Nicolai, and K. S. Stelle, "Universal BPS structure of stationary supergravity solutions," JHEP 07 (2009) 003, arXiv:0902.4438 [hep-th].

[10] G. Compere, S. de Buyl, E. Jamsin, and A. Virmani, "G2 Dualities in D=5 Supergravity and Black Strings," Class. Quant. Grav. 26 (2009) 125016, arXiv:0903.1645 [hep-th].

[11] W. Chemissany, J. Rosseel, M. Trigiante, and T. Van Riet, "The full integration of black hole solutions to symmetric supergravity theories," Nucl. Phys. B830 (2010) 391-413, arXiv:0903.2777 [hep-th].

[12] S. Bellucci, S. Ferrara, M. Gunaydin, and A. Marrani, "SAM Lectures on Extremal Black Holes in d=4 Extended Supergravity," arXiv:0905.3739 [hep-th].

[13] G. Bossard, "The extremal black holes of $\mathrm{N}=4$ supergravity from so( $8,2+\mathrm{n})$ nilpotent orbits," Gen. Rel. Grav. 42 (2010) 539-565, arXiv:0906.1988 [hep-th].

[14] M. Gunaydin, "Lectures on Spectrum Generating Symmetries and U-duality in Supergravity, Extremal Black Holes, Quantum Attractors and Harmonic Superspace," arXiv:0908.0374 [hep-th].

[15] G. Bossard, Y. Michel, and B. Pioline, "Extremal black holes, nilpotent orbits and the true fake superpotential," JHEP 01 (2010) 038, arXiv:0908.1742 [hep-th].

[16] S. Ferrara, A. Marrani, and E. Orazi, "Maurer-Cartan Equations and Black Hole Superpotentials in N =8 Supergravity," Phys. Rev. D81 (2010) 085013, arXiv:0911.0135 [Unknown].

[17] S.-S. Kim, J. L. Hornlund, J. Palmkvist, and A. Virmani, "Extremal solutions of the S3 model and nilpotent orbits of G2(2)," arXiv:1004.5242 [Unknown]. 
[18] P. Meessen, T. Ortin, S. Vaula, T. Ortin, and S. Vaula, "All the timelike supersymmetric solutions of all ungauged d=4 supergravities," arXiv:1006.0239 [Unknown].

[19] W. Chemissany et al., "Black holes in supergravity and integrability," arXiv:1007.3209 [Unknown].

[20] G. Compere, S. de Buyl, S. Stotyn, and A. Virmani, "A General Black String and its Microscopics," arXiv:1006.5464 [hep-th].

[21] S. Giusto and A. Saxena, "Stationary axisymmetric solutions of five dimensional gravity," Class. Quant. Grav. 24 (2007) 4269-4294, arXiv:0705.4484 [hep-th].

[22] A. Bouchareb et al., " $G_{2}$ generating technique for minimal $\mathrm{D}=5$ supergravity and black rings," Phys. Rev. D76 (2007) 104032, arXiv:0708.2361 [hep-th].

[23] J. Ford, S. Giusto, A. Peet, and A. Saxena, "Reduction without reduction: Adding KK-monopoles to five dimensional stationary axisymmetric solutions," Class. Quant. Grav. 25 (2008) 075014, arXiv:0708.3823 [hep-th].

[24] S. Giusto, S. F. Ross, and A. Saxena, "Non-supersymmetric microstates of the D1-D5-KK system," JHEP 12 (2007) 065, arXiv:0708.3845 [hep-th].

[25] D. V. Gal'tsov and N. G. Scherbluk, "Generating technique for $U(1)^{3} 5 D$ supergravity," Phys. Rev. D78 (2008) 064033, arXiv:0805.3924 [hep-th].

[26] J. Camps, R. Emparan, P. Figueras, S. Giusto, and A. Saxena, "Black Rings in Taub-NUT and D0-D6 interactions," JHEP 02 (2009) 021, arXiv:0811.2088 [hep-th].

[27] D. V. Gal'tsov and N. G. Scherbluk, "Three-charge doubly rotating black ring," Phys. Rev. D81 (2010) 044028, arXiv:0912.2771 [hep-th].

[28] M. Berkooz and B. Pioline, "5D Black Holes and Non-linear Sigma Models," JHEP 05 (2008) 045, arXiv:0802.1659 [hep-th].

[29] H. Elvang, R. Emparan, and P. Figueras, "Non-supersymmetric black rings as thermally excited supertubes," JHEP 02 (2005) 031, arXiv: hep-th/0412130.

[30] M. Cvetic and F. Larsen, "General rotating black holes in string theory: Greybody factors and event horizons," Phys. Rev. D56 (1997) 4994-5007, arXiv:hep-th/9705192.

[31] M. Cvetic and D. Youm, "General Rotating Five Dimensional Black Holes of Toroidally Compactified Heterotic String," Nucl. Phys. B476 (1996) 118-132, arXiv:hep-th/9603100.

[32] S. Giusto, S. D. Mathur, and A. Saxena, "Dual geometries for a set of 3-charge microstates," Nucl. Phys. B701 (2004) 357-379, arXiv:hep-th/0405017.

[33] V. Jejjala, O. Madden, S. F. Ross, and G. Titchener, "Non-supersymmetric smooth geometries and D1-D5-P bound states," Phys. Rev. D71 (2005) 124030, arXiv:hep-th/0504181. 
[34] O. J. C. Dias, R. Emparan, and A. Maccarrone, "Microscopic Theory of Black Hole Superradiance," Phys. Rev. D77 (2008) 064018, arXiv:0712.0791 [hep-th].

[35] J. C. Breckenridge, R. C. Myers, A. W. Peet, and C. Vafa, "D-branes and spinning black holes," Phys. Lett. B391 (1997) 93-98, arXiv:hep-th/9602065.

[36] G. W. Gibbons and C. A. R. Herdeiro, "Supersymmetric rotating black holes and causality violation," Class. Quant. Grav. 16 (1999) 3619-3652, arXiv:hep-th/9906098.

[37] P. Breitenlohner, D. Maison, and G. W. Gibbons, "Four-Dimensional Black Holes from Kaluza-Klein Theories," Commun. Math. Phys. 120 (1988) 295.

[38] D. Collingwood and W. McGovern, Nilpotent Orbits In Semisimple Lie Algebras. Van Nostrand Reinhold Mathematics series, 1993.

[39] D. Djokovic, "The closure diagrams for nilpotent orbits of real forms of $f_{4}$ and $g_{2}$," Journal of Lie Theory 10 (2000) 491-490.

[40] P. Figueras, E. Jamsin, J. V. Rocha, and A. Virmani, "Integrability of Five Dimensional Minimal Supergravity and Charged Rotating Black Holes," arXiv:0912.3199 [hep-th]. 